\title{
Benefits of Position-Sensitive Detectors for Radioactive Source Detection
}

\author{
Daniel J. Lingenfelter, Student Member, IEEE, Jeffrey A. Fessler, Fellow, IEEE, Clayton D. Scott, Member, IEEE, \\ and Zhong He, Senior Member, IEEE
}

\begin{abstract}
There are many systems for counting photons such as gamma-rays emitted from radioactive sources. Many of these systems are also position-sensitive, which means that the system provides directional information about recorded events. This paper investigates whether or not the additional information provided by position-sensitive capability improves the performance of detecting a point-source in background. We analyze the asymptotic performance of the generalized likelihood ratio test (GLRT) and a test based on the maximum-likelihood (ML) estimate of the source intensity for systems with and without position-sensitive capability. When the background intensity is known and detector sensitivity is spatially uniform, we prove that position-sensitive capability increases the area under the receiver operating characteristic curve (AUC). For cases when detector sensitivity is nonuniform or background intensity is unknown, we provide numerical results to illustrate the effect of the parameters on detection performance.
\end{abstract}

Index Terms-Asymptotic, detection, generalized likelihood ratio test (GLRT).

\section{INTRODUCTION}

$\mathbf{T}$ HE ability to detect radioactive material is important for security and nuclear nonproliferation. For security applications, the goal is to screen passengers and cargo for nuclear explosives or other potentially harmful materials. For nonproliferation applications, the goal is to assess the amount of different isotopes present at a nuclear reactor to ensure that the facility is not producing weapons materials. One means of detecting radioactive sources is to count gamma-ray photons emitted from it. Such a detector, hereafter called a counting detector, is limited by its inability to determine the direction or other attributes of incoming photons. In contrast, a position-sensitive detector provides some information in addition to the number of counts. It is reasonable to hypothesize that directional information can improve detection capability by distinguishing between photons

Manuscript received August 19, 2009; accepted May 12, 2010. Date of publication June 07, 2010; date of current version August 11, 2010. The associate editor coordinating the review of this manuscript and approving it for publication was Dr. Ta-Hsin Li. This work was supported by the U.S. Department of Homeland Security—DNDO under Grant Award 2008-DN-077-ARI007-02.

D. J. Lingenfelter, J. A. Fessler, and C. D. Scott are with the Department of Electrical Engineering and Computer Science, University of Michigan, Ann Arbor, MI 48109 USA (e-mail: danling@umich.edu; fessler@umich.edu; clayscot@umich.edu).

Z. He is with the Department of Nuclear Engineering and Radiological Sciences, University of Michigan, Ann Arbor, MI 48109 USA (e-mail: hezhong@umich.edu).

Color versions of one or more of the figures in this paper are available online at http://ieeexplore.ieee.org.

Digital Object Identifier 10.1109/TSP.2010.2051805 from a localized source and photons from a distributed background.

There is a wide body of literature on the problem of gamma-ray source detection. A common assumption in the field of security imaging is that sources of radiation are small, so they appear point-like to a detector [1]. There are many gamma-ray detection modalities including stationary radiation portal monitors [1], coded aperture imaging systems [2], arrays of scintillating detectors [3], and position-sensitive Compton detectors [4], [5]. Some detection algorithms are specific to certain modalities, such as using images reconstructed from a coded aperture system to detect a point-source [2]. A mean difference test (MDT) statistic was applied to scintillating arrays in [3]. In this paper, we analyze the detection performance of two tests that are based on the likelihood: one is the generalized likelihood ratio test (GLRT), and the other is the ML estimate of the source intensity. We refer to the latter as the source intensity test (SIT). We compare the asymptotic performance of the GLRT and SIT to the MDT presented in [3].

When performing detection using the GLRT with a position-sensitive Compton imaging detector in a known background, a particular experiment found that position information did not significantly improve detection performance over merely counting received photons [6]. In light of the numerous modalities that provide information beyond the number of received counts, we seek to show theoretically how position-sensitive capability affects detection performance. The goals of this work are to explain previous empirical results, such as those contained in [6], and to help guide the design of future detectors.

The question of whether or not imaging capability improves detection performance was addressed in [7] in the context of coded-aperture imaging systems. Reference [7] shows that if the background intensity is unknown, imaging may improve SNR [7] and thus detection performance by providing a means to separate the otherwise indistinguishable source and background photons. The analysis in this paper differs from that of [7] because we treat the problem from a detection task-based point of view [8]. We analyze the task of source detection using the asymptotic performance of various test statistics applied to detectors with and without position-sensitive capability. Our treatment of detection differs from that in [7] because SNR does not capture the additional information received on a per-photon basis by a position-sensitive detector.

In this work, we quantify detection performance using the area under the receiver operating characteristic curve (AUC). This metric is independent of any particular threshold value and is a measure of the overall detectability [9]. 
The novel contribution of this work is the task-based analysis of the asymptotic performance of the GLRT and SIT for detecting a single source in background using detectors with and without position-sensitive capability. We prove Theorem 1, which states that in a known background, a uniform-sensitivity position-sensitive detector always has equal or better detection performance asymptotically, in terms of AUC, than a counting detector of equal sensitivity. Our analysis also provides an expression that quantifies how much position-sensitive capability increases the AUC.

We compare the AUCs of position-sensitive and counting detectors when the assumptions of Theorem 1 do not hold. For the case of known background and nonuniform sensitivity, we numerically evaluate the asymptotic AUC of the GLRT and SIT for a simple position-sensitive detector of nonuniform sensitivity. Our results show that, unlike the uniform sensitivity case, a position-sensitive detector with nonuniform sensitivity can have a smaller AUC than a uniform-sensitivity counting detector of equal area for some source positions, particularly for a position-sensitive detector with poor sensitivity and poor position resolution. However, as sensitivity and resolution improve, the position-sensitive detector can have better detection performance. We use examples of various detectors to show how nonuniformity and other detector properties relate to detection performance, which could help in practical design problems.

We also show how relaxing the assumption of a known background impacts detection performance in terms of AUC through numerical examples. We use simple detectors to show that the degradation in detection performance caused by an unknown background is related to uncertainty in the source position estimate. The information that a position-sensitive detector records allows it to distinguish source and background photons when the source and background have different spatial distributions but identical energies. A counting detector does not have this capability when the source and background photons are of the same energy, so it is difficult to directly compare position-sensitive and counting detectors in an unknown background. More specifically, one cannot set the appropriate threshold for a test using a counting detector. In our analysis, we assume that the operator of a counting detector sets the threshold with a randomly chosen background intensity and examine how the distribution of the chosen background intensity affects the difference in AUC between the counting and position-sensitive detectors. The specific examples are meant to be a guide in analyzing and comparing particular systems.

\section{MATHEMATICAL BACKGROUND}

The model described in this section is general enough to describe any system that records a Poisson-distributed number of measurements or events, where the events are independent and each event is described by a vector of recorded attributes. This model accurately describes position-sensitive Compton detectors, coded aperture detectors, and scintillator arrays. The model is based on [8] and [10]. It assumes a fixed scan time, thus the number of recorded events is random.

\section{A. Measurement Model}

Following the notation of [8], let $\tilde{\boldsymbol{r}}=\left(\boldsymbol{r}_{1}, \boldsymbol{r}_{2}, \ldots, \boldsymbol{r}_{J}\right)$ denote a list of $J$ recorded attribute vectors from photon interaction events. Each element $\boldsymbol{r}_{i}$ of $\tilde{\boldsymbol{r}}$ is itself a vector of attributes describing the $i$ th event. An example of a detector that one can describe with this model is a position-sensitive Compton detector. A Compton detector records a Poisson-distributed number $J$ of gamma-ray photons. Each detected photon interacts one or more times inside the detector and the detector records these interaction locations and possibly other attributes such as deposited energy. We assume that distinct attribute vectors are statistically independent, which is reasonable provided the count rates are low enough to avoid dead time effects [8].

In the detection problem considered here, the goal is to decide whether or not a point-source is present in an environment with some background. Denote the source intensity by $\alpha \in[0, \infty)$ with units of counts emitted per unit time. The probability distribution of recorded attribute vectors $\boldsymbol{r}$ for events that originate at the source may depend on parameters other than the source intensity, and we denote the vector of additional parameters by $\phi \in \Phi$. In the 3-D far-field when the source and background are of the same energy, an example for the set $\Phi$ is $[0,2 \pi] \times[0, \pi]$, which represents all possible source positions in terms of azimuthal and polar angle in space. If a detector is energy-sensitive and the source and background energy spectra differ, the source energy could also be an element of $\phi$.

We model the background as a linear combination of a finite number of fixed, known distributions. We parameterize the background by the rate $\lambda_{b}$ in counts recorded per unit time and a vector of mixture coefficients $\boldsymbol{\beta}$, such that the probability distribution of recorded events given that they originated from background is a mixture of the distributions of recorded attributes given that they came from each object [10]. For this linear model, the background count rate, $\lambda_{b}$, is not a function of $\beta$.

We define the vector $\boldsymbol{\theta}$ to be the vector of all unknown parameters. When the source and background intensities and position parameters are all unknown,

$$
\boldsymbol{\theta}=\left(\alpha, \boldsymbol{\phi}, \lambda_{b}, \boldsymbol{\beta}\right) .
$$

A similar parameterization of a far-field point source is given in [3].

Let $D$ be the event that a photon is recorded and $G$ be the event that a photon passes through the detector. We define the sensitivity, which is the probability of recording a photon given that it came from direction $\phi$, to be

$$
s(\boldsymbol{\phi}) \triangleq \mathrm{p}(D ; \phi)=\mathrm{p}(D \mid G ; \phi) \mathrm{p}(G ; \boldsymbol{\phi}),
$$

where $\mathrm{p}(D \mid G ; \boldsymbol{\phi})$ is the intrinsic sensitivity, which depends on the detector shape and attenuation, and $\mathrm{p}(G ; \boldsymbol{\phi})$ is the geometric sensitivity, which depends on the fraction of emitted photons that pass through the detector [11, p. 65]. In 3-D, the geometric sensitivity is the solid angle subtended by the detector in a spherical coordinate system centered at the source.

Let $\mathrm{p}(\boldsymbol{r} \mid D ; \boldsymbol{\theta})$ denote the distribution of recorded attributes $r \in \mathcal{R}$, where $\mathcal{R}$ is the set of all possible event attributes. Let $\mathrm{p}_{S}(\boldsymbol{r} \mid D ; \boldsymbol{\phi})$ and $\mathrm{p}_{B}(\boldsymbol{r} \mid D ; \boldsymbol{\beta})$ denote the distributions of recorded 
event attributes $\boldsymbol{r}$ given that they are detected and come from the source and background, respectively. The overall distribution of recorded attributes (given that an event is detected) is a mixture of $\mathrm{p}_{S}(\boldsymbol{r} \mid D ; \boldsymbol{\phi})$ and $\mathrm{p}_{B}(\boldsymbol{r} \mid D ; \boldsymbol{\beta})$ given by [10]

$$
\mathrm{p}(\boldsymbol{r} \mid D ; \boldsymbol{\theta})=\frac{\lambda_{b} \mathrm{p}_{B}(\boldsymbol{r} \mid D ; \boldsymbol{\beta})+\alpha s(\boldsymbol{\phi}) \mathrm{p}_{S}(\boldsymbol{r} \mid D ; \boldsymbol{\phi})}{\lambda_{b}+\alpha s(\boldsymbol{\phi})} .
$$

As shown in [8], the likelihood of $\boldsymbol{\theta}$ is

$$
\mathrm{p}(\tilde{\boldsymbol{r}} ; \boldsymbol{\theta})=\prod_{i=1}^{J} \frac{\mathrm{p}\left(\boldsymbol{r}_{i} \mid D ; \boldsymbol{\theta}\right) e^{-\bar{J}(\boldsymbol{\theta})} \bar{J}(\boldsymbol{\theta})^{J}}{J !},
$$

and the number of recorded photons obeys the Poisson distribution

$$
J \sim \operatorname{Poisson}(\bar{J}(\boldsymbol{\theta}))
$$

with mean given by

$$
\bar{J}(\boldsymbol{\theta}) \triangleq \mathrm{E}[J]=\tau\left(\lambda_{b}+\alpha s(\phi)\right)
$$

where $\tau$ denotes the known measurement recording time.

We can make the concept of a counting detector more concrete by the following definition:

Definition 1: A detector is a counting detector if and only if $\mathrm{p}_{S}(\boldsymbol{r} \mid D ; \phi)=\mathrm{p}_{B}(\boldsymbol{r} \mid D ; \boldsymbol{\beta})$ almost everywhere ${ }^{1}$ for all $\boldsymbol{\phi} \in \Phi$ and mixture coefficients $\beta$.

Definition 1 says that in a counting detector, the distribution of event attributes is independent of whether or not the event originated from the source. Otherwise we call it an position-sensitive detector. By this definition, a detector that is energy-sensitive but not does not record interaction locations is not a counting detector. In such a detector, the recorded energies can produce some position information, so we treat spectrometers that do not record interaction locations similarly to spectrometers that do. The asymptotic expressions in this paper allow one to compare the asymptotic detection performance of particular spectrometers that do and do not record interaction locations. We refer to detectors that are not counting detectors as position-sensitive detectors, although a position-sensitive detector does not necessarily record interaction locations.

\section{B. Fisher Information}

The asymptotic detection performances of the test statistics examined in this work depend on the Fisher information matrix $F(\theta)$. To facilitate the analysis of $F(\boldsymbol{\theta})$, we define its block components as follows:

$$
\mathrm{F}(\boldsymbol{\theta})=\left[\begin{array}{llll}
\mathrm{F}_{[1,1]} & \mathrm{F}_{[2,1]}^{T} & \mathrm{~F}_{[3,1]}^{T} & \mathrm{~F}_{[4,1]}^{T} \\
\mathrm{~F}_{[2,1]} & \mathrm{F}_{[2,2]} & \mathrm{F}_{[3,2]}^{T} & \mathrm{~F}_{[4,2]}^{T} \\
\mathrm{~F}_{[3,1]} & \mathrm{F}_{[3,2]} & \mathrm{F}_{[3,3]} & \mathrm{F}_{[4,3]}^{T} \\
\mathrm{~F}_{[4,1]} & \mathrm{F}_{[4,2]} & \mathrm{F}_{[4,3]} & \mathrm{F}_{[4,4]}
\end{array}\right],
$$

where $\mathrm{F}_{[1,1]}$ is $1 \times 1, \quad \mathrm{~F}_{[2,1]}$ is $\operatorname{dim}(\phi) \times 1, \quad \mathrm{~F}_{[2,2]}$ is $\operatorname{dim}(\boldsymbol{\phi}) \times \operatorname{dim}(\boldsymbol{\phi}), \quad \mathrm{F}_{[3,1]}$ is $1 \times 1, \quad \mathrm{~F}_{[3,2]}$ is $1 \times \operatorname{dim}(\boldsymbol{\phi})$, $\mathrm{F}_{[3,3]}$ is $1 \times 1, \mathrm{~F}_{[4,1]}$ is $\operatorname{dim}(\boldsymbol{\beta}) \times 1, \mathrm{~F}_{[4,2]}$ is $\operatorname{dim}(\boldsymbol{\beta}) \times \operatorname{dim}(\boldsymbol{\phi})$, $\mathrm{F}_{[4,3]}$ is $\operatorname{dim}(\boldsymbol{\beta}) \times 1$, and $\mathrm{F}_{[4,4]}$ is $\operatorname{dim}(\boldsymbol{\beta}) \times \operatorname{dim}(\boldsymbol{\beta})$. In source detection problems, the source position $\phi$, the background in-

\footnotetext{
${ }^{1}$ Throughout, "almost everywhere" means with respect to the distribution of $r$ in (3)
}

tensity $\lambda_{b}$, and the background shape parameters $\beta$ are nuisance parameters.

A counting detector is neither capable of estimating $\phi$ nor distinguishing source and background events of the same energy because $\mathrm{p}_{S}(\boldsymbol{r} \mid D ; \boldsymbol{\phi})$ does not depend on $\phi$ by Definition 1 . Because of this, for the purposes of defining the Fisher information, we assume that $\lambda_{b}, \boldsymbol{\beta}$, and the value of $s(\boldsymbol{\phi})$ are known to a counting detector, so the Fisher information is a scalar in this case. Using the model in (4) and (5), the likelihood for the counting case is $\mathrm{p}(J ; \boldsymbol{\theta})=\bar{J}(\boldsymbol{\theta})^{J} e^{-\bar{J}(\boldsymbol{\theta})} / J$ !, for which the Fisher information is given by

$$
\mathrm{F}_{c}(\boldsymbol{\theta}) \triangleq \frac{\tau s^{2}(\boldsymbol{\phi})}{\lambda_{b}+\alpha s(\boldsymbol{\phi})} .
$$

To help express the Fisher information matrix (7) for a position-sensitive detector, we first define the following functions of $r$ :

$$
\begin{aligned}
& g_{1}(\boldsymbol{r})=\mathrm{p}_{S}(\boldsymbol{r} \mid D ; \boldsymbol{\phi}) \\
& g_{2}(\boldsymbol{r})=\nabla_{\boldsymbol{\phi}}\left(s(\boldsymbol{\phi}) \mathrm{p}_{S}(\boldsymbol{r} \mid D ; \boldsymbol{\phi})\right) \\
& g_{3}(\boldsymbol{r})=\mathrm{p}_{B}(\boldsymbol{r} \mid D ; \boldsymbol{\beta}) \\
& g_{4}(\boldsymbol{r})=\nabla_{\boldsymbol{\beta}} \mathrm{p}_{B}(\boldsymbol{r} \mid D ; \boldsymbol{\beta})
\end{aligned}
$$

where $\nabla_{\beta}$ is the column gradient with respect to $\beta$. Note that $g_{1}, g_{3}: \mathcal{R} \rightarrow \mathbb{R}, g_{2}: \mathcal{R} \rightarrow \mathbb{R}^{\operatorname{dim}(\boldsymbol{\phi})}$, and $g_{4}: \mathcal{R} \rightarrow \mathbb{R}^{\operatorname{dim}(\boldsymbol{\beta})}$. Using (3)-(5), one can show that the Fisher Information for a position-sensitive detector is given by

$$
\mathrm{F}(\boldsymbol{\theta})=\mathrm{F}_{\mathrm{C}}(\boldsymbol{\theta})\left[\begin{array}{cccc}
K_{[1,1]} & \frac{\alpha K_{[2,1]}^{T}}{s(\boldsymbol{\phi})} & \frac{K_{[3,1]}}{s(\boldsymbol{\phi})} & \frac{\lambda_{b} K_{[4,1]}^{T}}{s(\boldsymbol{\phi})} \\
\frac{\alpha K_{[2,1]}}{s(\boldsymbol{\phi})} & \frac{\alpha^{2} K_{[2,2]}}{s^{2}(\boldsymbol{\phi})} & \frac{\alpha K_{[3,2]}^{T}}{s^{2}(\boldsymbol{\phi})} & \frac{\alpha \lambda_{b} K_{[4,2]}^{T}}{s^{2}(\boldsymbol{\phi})} \\
\frac{K_{[3,1]}}{s(\boldsymbol{\phi})} & \frac{\alpha K_{[3,2]}}{s^{2}(\boldsymbol{\phi})} & \frac{K_{[3,3]}}{s^{2}(\boldsymbol{\phi})} & \frac{\lambda_{b} K_{[4,3]}^{T}}{s^{2}(\boldsymbol{\phi})} \\
\frac{\lambda_{b} K_{[4,1]}}{s(\boldsymbol{\phi})} & \frac{\alpha \lambda_{b} K_{[4,2]}}{s^{2}(\boldsymbol{\phi})} & \frac{\lambda_{b} K_{[4,3]}}{s^{2}(\boldsymbol{\phi})} & \frac{\lambda_{b}{ }^{2} K_{[4,4]}}{s^{2}(\boldsymbol{\phi})}
\end{array}\right],
$$

where

$$
K_{[i, j]} \triangleq \mathrm{E}\left[\frac{g_{i}(\boldsymbol{r}) g_{j}^{T}(\boldsymbol{r})}{\mathrm{p}^{2}(\boldsymbol{r} \mid D ; \boldsymbol{\theta})}\right],
$$

provided that the expectation and the gradient with respect to the parameters are interchangeable. Appendix B gives sample derivations of the block Fisher information elements.

In the case where the background intensities are known, we remove the entries corresponding to the unknown background and the Fisher information for a position-sensitive detector simplifies to

$$
\mathrm{F}(\boldsymbol{\theta})=\mathrm{F}_{\mathrm{c}}(\boldsymbol{\theta})\left[\begin{array}{cc}
K_{[1,1]} & \frac{\alpha}{s(\boldsymbol{\phi})} K_{[2,1]}^{T} \\
\frac{\alpha}{s(\boldsymbol{\phi})} K_{[2,1]} & \frac{\alpha^{2}}{s^{2}(\boldsymbol{\phi})} K_{[2,2]}
\end{array}\right] .
$$

The elements of the Fisher information appear prominently in the detection analyses that follow.

\section{Test Statistics for Source Detection}

In the source detection problem, we would like to discern between two hypotheses:

$$
\begin{aligned}
& H_{0}: \alpha=0 \\
& H_{1}: \alpha>0 .
\end{aligned}
$$


Based on the model (3) and (5), there are several reasonable tests for this problem.

1) Generalized Likelihood Ratio Test (GLRT): The GLRT is a common method of choosing between two hypotheses when one or more of the hypotheses depends on unknown parameters [12]. We can write the GLRT as

$$
2 \log \Lambda_{\mathrm{GLRT}} \underset{H_{0}}{\stackrel{H_{1}}{\gtrless}} \gamma,
$$

where the GLRT test statistic is

$$
\Lambda_{\mathrm{GLRT}} \triangleq \frac{\max _{\alpha, \boldsymbol{\phi}, \lambda_{b}, \boldsymbol{\beta}} \mathrm{p}\left(\tilde{\boldsymbol{r}} ;\left(\alpha, \boldsymbol{\phi}, \lambda_{b}, \boldsymbol{\beta}\right), H_{1}\right)}{\max _{\boldsymbol{\phi}, \lambda_{b}, \boldsymbol{\beta}} \mathrm{p}\left(\tilde{\boldsymbol{r}} ;\left(\alpha=0, \boldsymbol{\phi}, \lambda_{b}, \boldsymbol{\beta}\right), H_{0}\right)} .
$$

To calibrate the test threshold $\gamma$ and analyze the performance of the detector, one must determine the distribution of $\Lambda_{\mathrm{GLRT}}$. If one uses a restricted ML estimator (MLE) motivated by the onesided hypothesis in (12), the distribution of $\Lambda_{\mathrm{GLRT}}$ is complicated because under $H_{0}$, the parameter $\alpha$ lies on the boundary of the parameter space [13]. To simplify the analysis of the GLRT and give intuition, instead of (12), we consider the two-sided test

$$
\begin{aligned}
& H_{0}: \alpha=0 \\
& H_{1}: \alpha \neq 0,
\end{aligned}
$$

which is also the basis of the analysis in [14]. When the test is treated with the two-sided formulation in (15) and the source intensity is small, it is shown in [12, pp. 239-240], that the asymptotic distribution of the test statistic is given by

$$
2 \log \left(\Lambda_{\mathrm{GLRT}}\right) \sim \begin{cases}\chi_{1}^{2}(\eta), & H_{1} \\ \chi_{1}^{2}(0), & H_{0},\end{cases}
$$

where $\chi_{1}^{2}(\eta)$ denotes the noncentral chi-square distribution with one degree of freedom and noncentrality parameter $\eta$. Assuming $F(\theta)$ is invertible, for the model (4), the noncentrality parameter is

$$
\eta=\alpha^{2}\left(\mathrm{~F}^{-1}(\boldsymbol{\theta})_{[1,1]}\right)^{-1} .
$$

This asymptotic framework is also used to analyze a test of whether one or two sources are present in [14].

2) Wald Test: The Wald test is a classical test for the composite hypothesis problem and is known to be approximately asymptotically equivalent to the GLRT for small source intensities [12, p. 188]. The Wald test for the source detection problem is given by

$$
\Lambda_{\mathrm{W}}=\hat{\alpha}_{\mathrm{ML}}^{2}\left(\mathrm{~F}^{-1}(\hat{\boldsymbol{\theta}})_{[1,1]}\right)^{-1},
$$

where $\hat{\boldsymbol{\theta}}$ is the ML estimate of $\boldsymbol{\theta}$. The Wald test is a nonmonotonic function of $\hat{\alpha}_{\mathrm{ML}}$ when $\hat{\alpha}_{\mathrm{ML}}$ is the unrestricted MLE of $\alpha$. Although the source intensity cannot be physically less than zero, one can find $\hat{\alpha}_{\mathrm{ML}}$ by maximizing the likelihood over the real line. Intuitively, a negative estimate of the source intensity is strong evidence for the null hypothesis. Either squaring or thresholding negative source intensity estimates at zero can re-

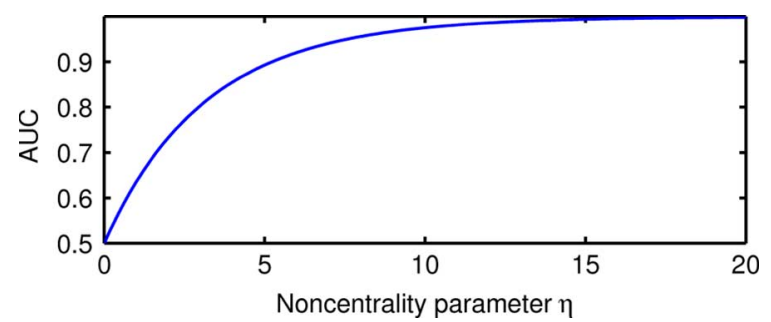

Fig. 1. AUC of GLRT (14) versus noncentraltity parameter $\eta$ of the asymptotic distribution of the GLRT.

duce detection performance by reducing the separability of the test statistic distributions under the two hypotheses, especially for weak sources. Our numerical results do not include the Wald test since it is asymptotically equivalent to the GLRT.

3) Source Intensity Test (SIT): Because the squaring operation in the Wald test statistic can degrade detection performance, we consider the following source intensity test statistic

$$
\Lambda_{\mathrm{SIT}} \triangleq \hat{\alpha}_{\mathrm{ML}}
$$

This test was also considered in the context of array processing [15]. By the asymptotic normality of the MLE [12, p. 240], we have that, asymptotically

$$
\hat{\alpha}_{\mathrm{ML}} \sim \begin{cases}\mathcal{N}\left(0, \mathrm{~F}^{-1}\left(\boldsymbol{\theta}_{0}\right)_{[1,1]}\right), & H_{0} \\ \mathcal{N}\left(\alpha, \mathrm{F}^{-1}(\boldsymbol{\theta})_{[1,1]}\right), & H_{1},\end{cases}
$$

where $\boldsymbol{\theta}_{0}$ is the parameter vector under the null hypothesis with $\alpha=0$. The next section uses the asymptotic distributions in (16) and (19) to show that position-sensitive capability improves detection performance.

4) Mean Difference Test (MDT): The mean difference test statistic is proposed in [3] and is equivalent to

$$
\Lambda_{\mathrm{MDT}} \triangleq J_{\mathrm{src}}-J_{\mathrm{back}}
$$

where $J_{\mathrm{src}}$ is the number of photons recorded on all source-exposed surfaces of the detector and $J_{\text {back }}$ is the number of photons recorded on all surfaces not exposed to the source. When the source position is unknown, one must estimate which surfaces are exposed to the source. The asymptotics of the MDT are given in [3].

\section{EFfect of Position-Sensitive CAPABILITy on DETECTION PERFORMANCE}

As illustrated in Fig. 1, the AUC of the GLRT for (15) is a monotone function of the noncentrality parameter $\eta$ in (17), so to show that position-sensitive capability improves AUC, it suffices to show that position-sensitive capability increases $\eta$. Furthermore, if position-sensitive capability increases $\eta$ for all values of $\alpha \geq 0$, then position-sensitive capability will improve the performance of the SIT by reducing the variance of the test statistic under each hypothesis in (19).

\section{A. Effect of Position-Sensitive Capability for a Uniform Sensitivity Detector With Known Background}

We first define the concept of a uniform-sensitivity detector: 
Definition 2: A detector has uniform-sensitivity if $s(\boldsymbol{\phi})=s_{0}$ for all $\phi \in \Phi$, where $s_{0}$ is a constant.

The main result of this section, given by Theorem 1, is that the detection performance of a uniform-sensitivity position-sensitive detector is greater than or equal to that of a uniform sensitivity counting detector. The AUC of the GLRT applied to a position-sensitive detector is greater than the AUC of the GLRT applied to a counting detector if the noncentrality parameter of the asymptotic distribution under $H_{1}$ in (17) is larger for a position-sensitive detector. As shown in [12, p. 232], the asymptotic distribution of the GLRT is most accurate for small source intensities because the derivation of the asymptotic distribution assumes that the $\log$-likelihood evaluated at $\alpha=0$ is approximately equal to the second order approximation of the log-likelihood about the value of the source intensity estimate $\hat{\alpha}_{\mathrm{ML}}$. For the SIT, a detector with position-sensitive capability performs better if the variances in (19) are smaller for the position-sensitive detector. The asymptotics of the SIT do not assume a small source intensity. The above discussion leads to the sufficient condition that position-sensitive capability improves the AUC of the GLRT and SIT for any $\boldsymbol{\theta} \in \boldsymbol{\Theta}$ :

$$
\left(\mathrm{F}(\boldsymbol{\theta})_{[1,1]}^{-1}\right)^{-1}>\mathrm{F}_{\mathrm{c}}(\boldsymbol{\theta})
$$

Theorem 1: For a uniform-sensitivity detector in a known background, $\left(\mathrm{F}^{-1}(\boldsymbol{\theta})_{[1,1]}\right)^{-1} \geq \mathrm{F}_{\mathrm{c}}(\boldsymbol{\theta})$, i.e., the reciprocal of the $[1,1]$ component of the inverse Fisher Information Matrix (11) for a position-sensitive detector is greater than or equal to that of a counting detector (8). Therefore, the asymptotic AUC for a position-sensitive detector is greater than or equal to the asymptotic AUC of a counting detector in a known background when the GLRT with a small source intensity or the SIT is used.

The proof, which is given in Appendix A, shows that the noncentrality parameters of the GLRT for position-sensitive and counting detectors, $\eta_{i}$ and $\eta_{c}$, respectively, obey

$$
\frac{\eta_{i}}{\eta_{c}}=\frac{\left(\mathrm{F}^{-1}(\boldsymbol{\theta})_{[1,1]}\right)^{-1}}{\mathrm{~F}_{\mathrm{c}}(\boldsymbol{\theta})} \geq 1
$$

Furthermore, if $\mathrm{F}^{-1}(\boldsymbol{\theta})$ is diagonal,

$$
\frac{\eta_{i}}{\eta_{\mathrm{c}}}=K_{[1,1]}
$$

so position-sensitive capability increases the noncentrality parameter $\eta$ in (17) by the factor $K_{[1,1]}$ in (9). In this case, the inequality in Theorem 1 becomes strict.

\section{SETUP fOR Numerical CALCUlations}

In Section V, we numerically evaluate the Fisher information (9) for 2-D circular detectors of radius $r$ and 2-D square detectors with side length $2 l$ to illustrate Theorem 1 and to explore the cases where Theorem 1 does not apply. The detectors in this section do not necessarily represent any particular detector technology and are used for illustrative purposes only. The simplified detectors have tractable models for the recorded attributes, facilitating accurate calculation of the Fisher information.

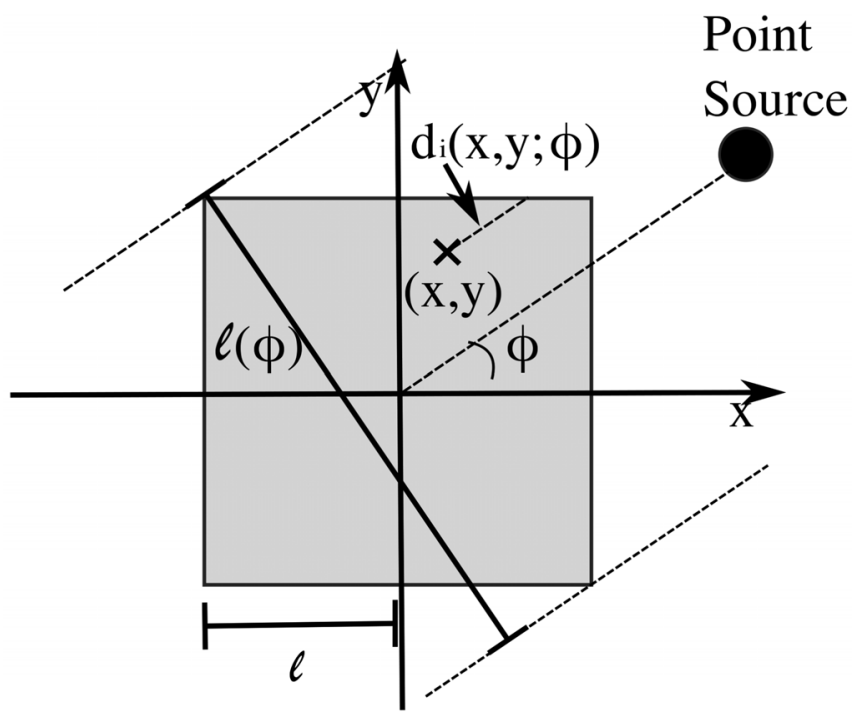

Fig. 2. Diagram of square detector with side length $2 l$.

For simplicity, we assume that these detectors record only single photon interaction events and that the source and background energy spectra are identical. For each recorded event, the detector records the position of the interaction $(x, y)$. The attribute vector $\boldsymbol{r}_{i}$ is the interaction position of the $i$ th event. Each attribute vector $\boldsymbol{r}_{i}$ has length 2, so we compute the components of the Fisher information (10) numerically using Riemann approximation. This hypothetical system allows us to gain intuition on how detector nonuniformity and unknown background affect detection performance. This intuition will be useful when thinking about more realistic 3-D detectors.

For this analysis, we assume that the point-source is in the far-field, so $\phi \in[0,2 \pi)$ denotes the source position in the 2-D plane. The density of recorded attributes, $\mathrm{p}(x, y \mid D ; \boldsymbol{\theta})$ depends on the source position $\phi$, so the parameter vector $\phi$ in (1) is equal to $\phi$.

The probability density of photon interaction locations is governed by the Beer-Lambert law for attenuation [16, pp. 54-56]. The density of interaction events at a particular point inside the detector is a decreasing function of the length of material that a photon must pass through to reach that point. The number of photons, on average, that interact in a given length of material is parameterized by $\mu$, the material linear attenuation coefficient at the energy of incoming gamma-rays, which is assumed to be known. The attenuation coefficient depends on detector material and influences its position resolution. The interaction probability distribution is given by

$$
\mathrm{p}(x, y \mid D ; \boldsymbol{\theta})=\frac{1}{l(\phi) \mathrm{p}(D ; \boldsymbol{\phi})} \mu e^{-\mu d_{i}(x, y ; \phi)} \quad(x, y) \in \mathcal{R},
$$

where $d_{i}(x, y ; \phi)$ is the distance that the photon must travel in the direction $\phi$ through the detector before interacting at $(x, y)$, and $l(\phi)$ is the largest distance between any two lines with slope $\tan (\phi)$ that pass through the detector. These quantities are illustrated in Fig. 2. 
It is difficult to define the intrinsic position resolution of these systems, so we report the angular uncertainty measured by the square root of the Cramér-Rao lower bound on the position estimate $\sqrt{\left(F(\boldsymbol{\theta})^{-1}\right)_{[2,2]}}$, where $F(\boldsymbol{\theta})$ is defined in (9).

\section{NUMERICAL RESULTS}

We first consider uniform-sensitivity position-sensitive and counting detectors in a known background to illustrate the results of Theorem 1 and to explore under what conditions position information is most beneficial. Next, we consider a square position-sensitive detector, which has nonuniform sensitivity, and compare its performance to a uniform counting detector of equal area. We then examine the performance of uniform-sensitivity detectors in unknown background. We exclude the case of a nonuniform sensitivity detector in an unknown background because its analysis requires many assumptions.

\section{A. Uniform Sensitivity Detector With Known Background}

A circular detector with radius $r$ has inherent uniform sensitivity due to its circular symmetry. Fig. 3(a) shows the AUC, the position-sensitive gain factor $K_{[1,1]}$ from (9), and the angular uncertainty as a function of attenuation-radius product $\mu r$ for a circular detector. The quantity $\alpha \tau$ represents the expected number of photons emitted from the source during the scan and $\lambda_{b} \tau$ represents the expected number of background photons recorded. The expected number of source photons recorded is given by $\alpha \tau s_{0}$, where $s_{0}=s(\boldsymbol{\phi})$ as defined in (2). We denote the sensitivity by $s_{0}$ to emphasize that the sensitivity of a circular detector is not a function of source position in the far field. In this section, we consider only source photons that pass through the detector, i.e., $\mathrm{p}(G ; \phi)=1$ in (2). As guaranteed by Theorem 1, the AUC of the position-sensitive detector always exceeds that of the counting detector for each test.

The AUC of the SIT exceeds that of the GLRT for this experiment. This performance difference comes from the fact that the GLRT is asymptotically equivalent to the Wald test, which involves a square of the source activity estimate. When one uses an unrestricted MLE of the source intensity, this squaring operation reduces the separation between test statistic values under the two hypotheses.

Fig. 3(d) shows $s_{0}$ as a function of $\mu r$ to aid interpreting Fig. 3(a). For this experiment, the Fisher information matrix is diagonal, so $K_{[1,1]}$ is the multiplicative improvement in the noncentrality parameter of the asymptotic distribution of the GLRT under $H_{1}$, as expressed in (23). As $\mu r$ increases, the angular uncertainty decreases and the difference in AUC between the detectors with and without position-sensitive information increases. Fig. 3(a) and (b) illustrates that as the detector provides more precise position-sensitive information, the improvement in detection performance due to position-sensitive information increases even when the background is known.

Fig. 4 shows the AUC for a circular uniform-sensitivity detector as a function of source intensity for a fixed background intensity. The AUC values for the position-sensitive and counting detectors differ the most for source intensities near the background intensity. For low source-to-background ratios, the source is difficult to detect with either detector, and when the source-to-background ratio is large, the source is so

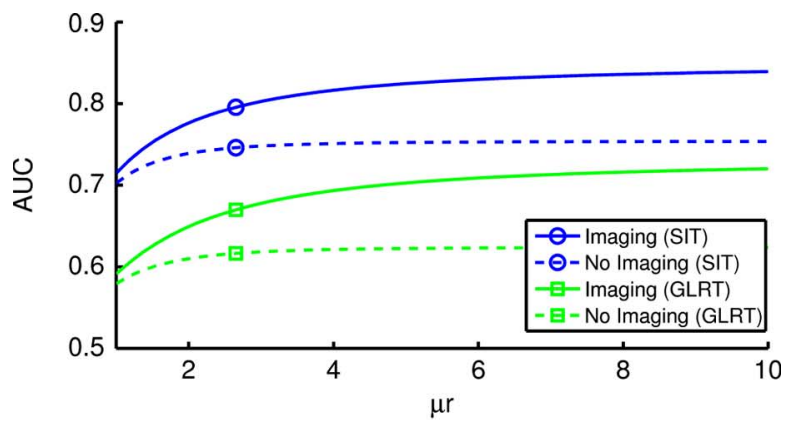

(a)

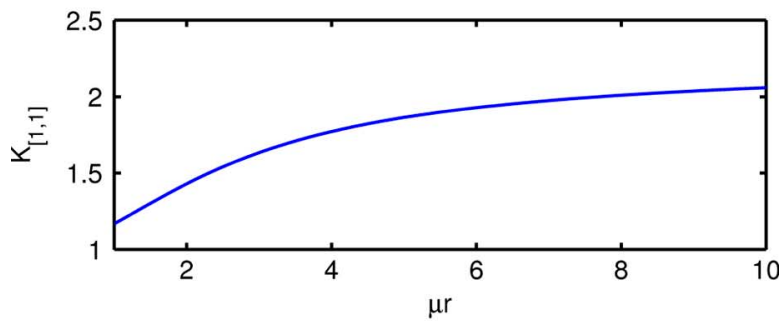

(b)

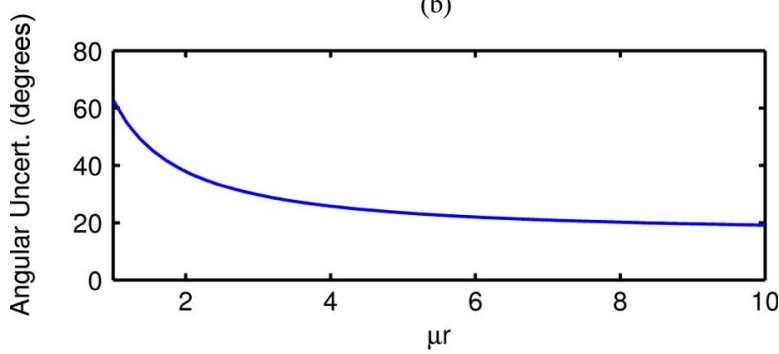

(c)

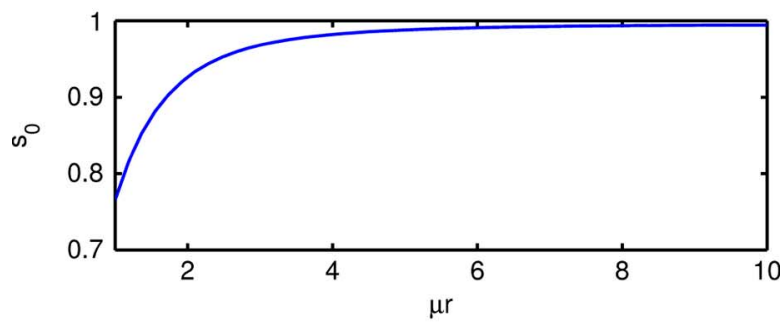

(d)

Fig. 3. Various quantities for a circular uniform-sensitivity detector with $\alpha \tau=10, \lambda_{b} \tau=100$, and geometric sensitivity $\mathrm{p}(G \mid \phi)=1$. (a) AUC versus $\mu r$; (b) $K_{[1,1]}$ versus $\mu r$; (c) angular uncertainty versus $\mu r$; and (d) $s_{0}$ versus $\mu r$.

easily detected that position-sensitive capability provides little additional benefit. Again, the position-sensitive detector always has higher AUC than the counting detector as expressed in Theorem 1, and the SIT performs better than the GLRT.

The next section considers the detection performance of nonuniform-sensitivity detectors, to which Theorem 1 does not apply, but which can be more practical to build than uniform sensitivity detectors.

\section{B. Nonuniform Sensitivity Detector With Known Background}

For implementation and manufacturing reasons, current position-sensitive Compton imaging detectors are often box-shaped [4]. We examined the performance of the GLRT and SIT applied to the 2-D square detector in Fig. 2 to gain insight into the performance of nonuniform sensitivity detectors. 


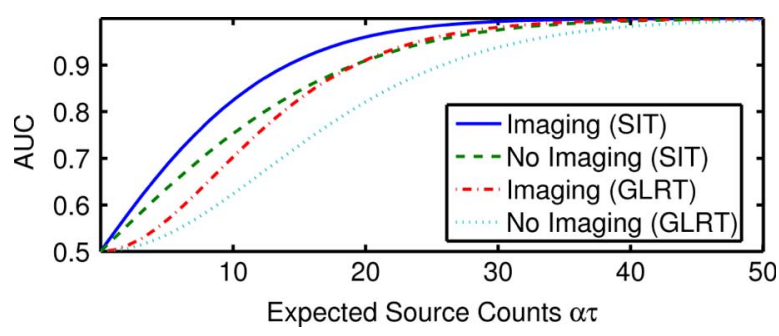

Fig. 4. AUC versus expected source counts for a circular uniform-sensitivity detector with $\lambda_{b} \tau=100$ and $\mu r=5$.

In the case of a position-sensitive detector, the maximumlikelihood estimate of the source intensity depends on $s(\boldsymbol{\phi})$, but a counting detector, by definition, gives no information about $\phi$. To compute the ML estimate of the source intensity with a counting detector, one would need to assume a particular value of $s(\phi)$ because $s(\phi)$ appears in the likelihood and is a function of the unobservable position $\phi$. Substituting a fixed value for $s(\phi)$ will result in a likelihood model that does not match the true distribution, so the estimator based on that model can be biased. Since the asymptotic analysis considered in this work does not apply when the parameter estimators are biased, we compare the square position-sensitive detector to a circular uniform-sensitivity counting detector of equal area with radius $r=2 l / \sqrt{\pi}$. This comparison constrains the amount of detector material and explores whether the position-sensitive square or the counting circle has better detection performance using the SIT and GLRT.

Fig. 5(a) shows the AUC of the square position-sensitive and the circular counting detectors as a function of source position for both the GLRT and SIT. For $\mu l=0.5$, the square position-sensitive detector performs worse than the circular counting detector. However, the detector with $\mu l=5$ outperforms the counting detector for all source positions. As in the uniform-sensitivity case, the SIT outperforms the GLRT.

To aid in visualization, we present the sensitivity of the square relative to that of a circular detector of equal area. To compute the relative sensitivity in Fig. 5(b), we normalized the geometric sensitivity of the square detector in (2) by that of a circular detector of the same area, so in this case,

$$
\mathrm{p}(G \mid \phi)=\frac{2 l(|\cos \phi|+|\sin \phi|)}{\frac{4 l}{\sqrt{\pi}}} .
$$

By using the relative sensitivity instead of the absolute sensitivity, the source intensity $\alpha$ has units of counts impinging on the detector per unit time. This figure, along with Fig. 5(a), shows that the detection performance of the square is better when the sensitivity is larger, and that detection performance is better for source positions where the slope of the sensitivity curve is small. In practice, one could generate similar plots with the appropriate sensitivity for a particular application.

Fig. 5(c) shows the angular uncertainty as a function of source position. The AUC in Fig. 5(a) is largest when the angular uncertainty is largest near $\phi=45^{\circ}$, but this is not contradictory because the sensitivity, shown in Fig. 5(b), is approximately uniform near $\phi=45^{\circ}$. Because of this, the Fisher information matrix is approximately diagonal for source
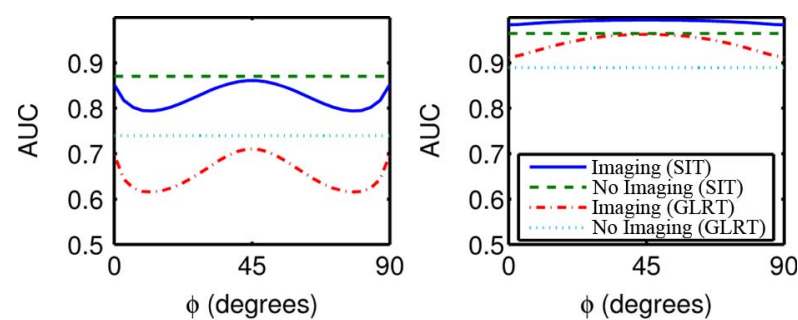

(a)
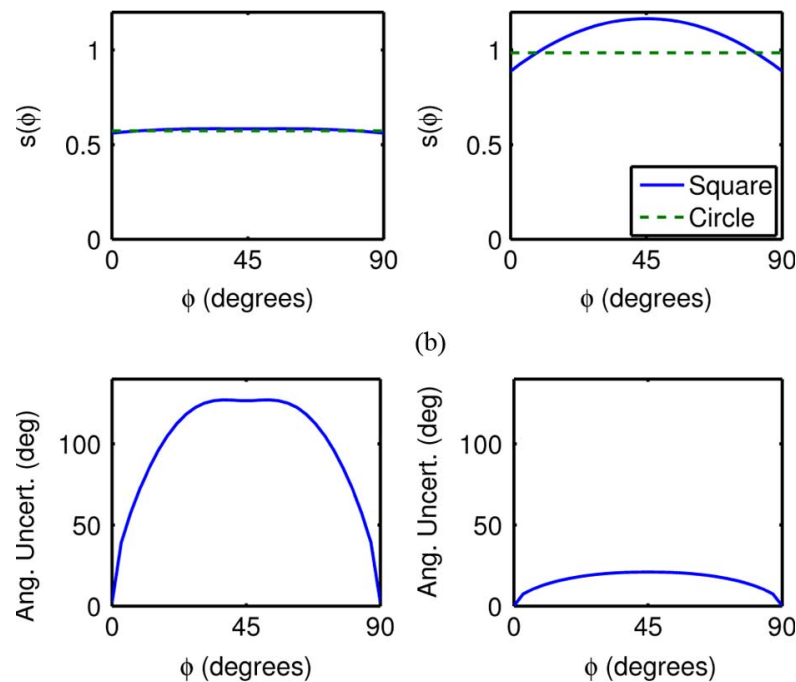

(c)

Fig. 5. AUC, relative sensitivity, and angular uncertainty for square positionsensitive and circular counting detectors of equal area $(r=2 l / \sqrt{\pi})$ versus $\phi$ with $\mu l=0.5$ (left) and $\mu l=5$ (right), and $\alpha \tau=\lambda_{b} \tau=10$. (a) AUC versus $\boldsymbol{\phi}$; (b) $s(\phi)$ versus $\boldsymbol{\phi}$; and (c) angular uncertainty versus $\boldsymbol{\phi}$.

positions near $\phi=45^{\circ}$, which means that the position nuisance parameter has little effect on the $[1,1]$ component of $F(\boldsymbol{\theta})$.

Whether a nonuniform sensitivity position-sensitive detector is better than a counting detector depends on the characteristics of the counting detector used for comparison. A position-sensitive detector provides information about the source position, whereas a counting detector does not. For some applications, the position information could outweigh a smaller AUC for some source positions.

\section{Uniform Sensitivity Detector With Spatially-Uniform Unknown Background}

We analyze the detection performance of a 2-D uniform-sensitivity circular position-sensitive detector with attenuation $\mu$ and radius $r$ with a point source in a spatially uniform background of unknown intensity $\lambda_{b}$, with the goal of examining how not knowing the background affects detection performance. We use the $[1,1]$ element of the inverse of the $3 \times 3$ block Fisher information matrix in (9). We remove the 4 th row and column of $F(\theta)$ because there is no $\beta$ to parameterize the background mixture in this model. We then use (16) and (19) to quantify the AUC.

Fig. 6 shows the AUC for a position-sensitive detector in a uniform background of known and unknown intensity as a function of the true background intensity. As the true background intensity increases, the difference between the AUCs of 


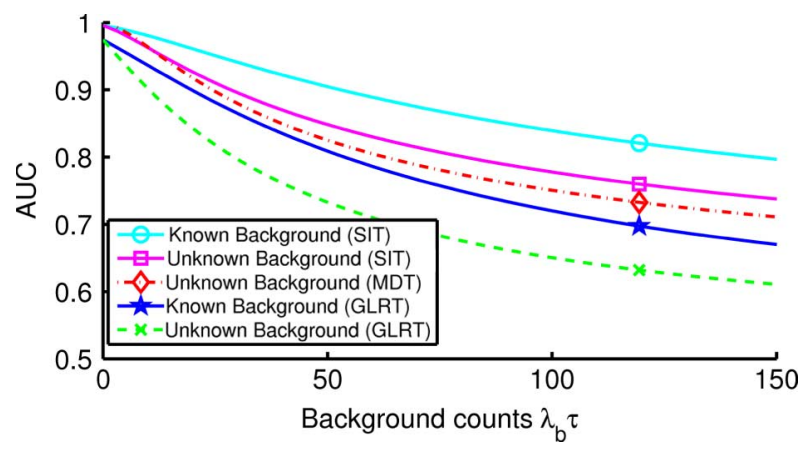

Fig. 6. AUC versus $\lambda_{b} \tau$ for spatially uniform background of known and unknown intensity background where $\mu r=10$ and $\alpha \tau=10$.

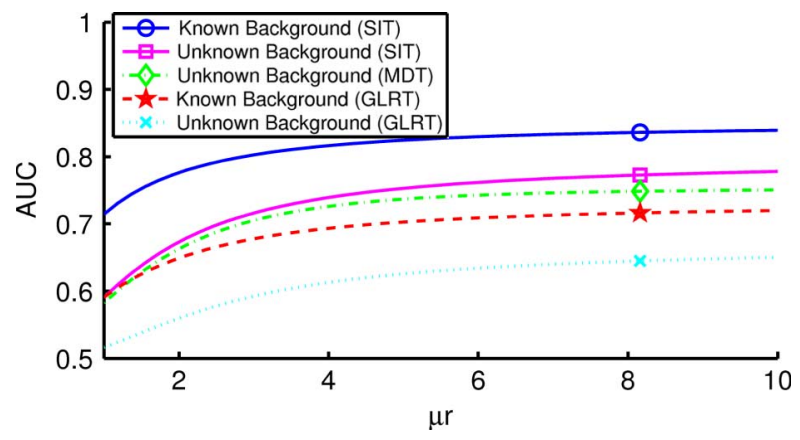

Fig. 7. AUC for spatially uniform background of known and unknown intensity versus attenuation-radius product for $\alpha \tau=10, \lambda_{b} \tau=100$.

known and unknown background increases for each particular test statistic. As in the known background case, the SIT outperforms the GLRT. In Fig. 6, we also show the performance of the MDT [3]. The MDT, as presented in [3] assumes a spherical array of detectors, and the detectors considered in this work record interactions in the interior of the detector. As the attenuation coefficient $\mu$ of these detectors increases, they behave more like a circular array of scintillators. To evaluate the performance of the MDT for finite-attenuation detectors, we computed the asymptotic mean number of counts recorded in the semicircles facing and opposite the source. The MDT performs better than the GLRT and worse than the SIT for low source-to-background ratios in this experiment. We assumed that the MDT knows the source position, so that the boundary between the two semicircles is not estimated. Although this is not a fair comparison, it gives the MDT the advantage.

Fig. 7 shows the AUC for a position-sensitive detector in a spatially uniform background of known and unknown intensity as a function of the attenuation-radius product. As the $\mu r$ product increases, the difference in AUC between the known and unknown background case decreases for the SIT and GLRT. Also, as $\mu r$ increases, the SIT performs better than the MDT. A possible explanation for this is that the SIT considers the interaction location of each photon and the MDT considers only the semicircle in which the interaction occurred.

Notice that the difference in AUC between the known and unknown background cases for each test statistic decreases as $\mu r$ increases. Recall from Fig. 3(c) that the angular uncertainty decreases as $\mu r$ increases. As the angular uncertainty decreases, the variance of the background intensity estimate decreases

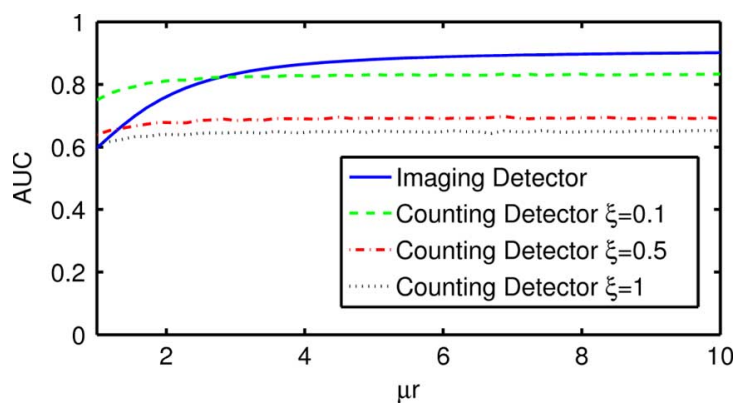

Fig. 8. AUC versus $\mu r$ for position-sensitive and counting detectors, where the counting detector uses a "guessed" background rate in the "known background" GLRT. $\alpha \tau=\lambda_{b} \tau=10$.

because the detector can more reliably distinguish source and background photons. One could recover the AUC "lost" by not knowing the background by increasing the scan time somewhat.

\section{Position-Sensitive Versus Counting Detectors With Unknown Background}

In practice, one can sometimes measure the background prior to screening for sources of interest. Also, an experienced operator of a counting detector could plausibly guess the background with some degree of uncertainty. The detection performance of such an operator depends on the accuracy or distribution of such guesses. As a hypothetical example, suppose that the operator of a counting detector applies the GLRT for a "known background" hypothesis test using a background rate $\check{\lambda}_{b}$ distributed according to the following gamma distribution [17, p. 291]:

$$
\mathrm{p}\left(\check{\lambda}_{b} ; \lambda_{b}, \xi\right)=\check{\lambda}_{b}^{\lambda_{b} / \xi-1} \frac{e^{-\check{\lambda}_{b} / \xi}}{\Gamma\left(\frac{\lambda_{b}}{\xi}\right) \xi^{\lambda_{b} / \xi}},
$$

where $\xi$ is a scale parameter that could represent the operator's accuracy and $\lambda_{b}$ is the true background rate. Note that $\mathrm{E}\left[\check{\lambda}_{b}\right]=\lambda_{b}$ and $\operatorname{Var}\left(\check{\lambda}_{b}\right)=\lambda_{b} \xi$. If $\xi$ is small, then the operator's guesses are narrowly distributed about the true background rate, and as $\xi$ increases, the guesses are farther from the mean, on average. The gamma distribution is a reasonable model for operator uncertainty because of its nonnegativity, and there may be other models that are more accurate in practice. Fig. 8 shows the AUC of the GLRT using an operator's guess, for various scale parameters $\xi$. Even when $\xi$ is small, the position-sensitive detector with large $\mu r$ still performs better. This is because as $\xi \rightarrow 0$ and $\mu r \rightarrow \infty$, the AUCs of both detectors approach the known background case, where Theorem 1 applies.

\section{CONCLUSION AND FutURE WORK}

We investigated how position-sensitive capability impacts the detection performance in photon counting detectors. In the case of a uniform-sensitivity detector in known background, we showed in Theorem 1 that position-sensitive capability always improves detection performance in terms of asymptotic AUC for the SIT and the GLRT when the source intensity is small relative to the background intensity. We also showed empirically that the SIT can outperform the GLRT in terms of AUC. 
In reality, detector sensitivity may be nonuniform. For nonuniform-sensitivity detectors, the benefit of position-sensitive capability depends on detector quality and the method of comparison. For sufficiently large $\mu l$, the square position-sensitive detector outperformed a round counting detector of equal area, but for small $\mu l$, the counting detector performed better for some source positions. Also, for sufficiently high $\mu l$, the position-sensitive detector can even outperform a hypothetical counting detector that "knows" the source position.

An unknown background intensity is likely in practical detection scenarios. As in the nonuniform sensitivity case, it is difficult to compare position-sensitive and counting detectors. We found that a position-sensitive detector that does not know the background rate $\lambda_{b}$ can outperform a counting detector with an operator who has the ability to estimate the background intensity accurately. However, the results in the unknown background case are mixed because which type of detector has a higher AUC depends on many factors, such as detector size, detector attenuation, and the particular background model.

To compare a nonuniform position-sensitive detector to a counting detector in the case of an unknown background, one must make assumptions about the operator of a counting detector, as we did with the uniform-sensitivity detector in unknown background. Alternatively, one could model the background intensity with a prior distribution. The particular application of the detection system will determine the best method of comparison. Detection performance in this case is governed by the combined effects of unknown background and nonuniform sensitivity, which we studied separately. The intuition from the separate analyses applies to the case of a nonuniform detector in an unknown background.

This work addressed the benefits of position-sensitive capability in photon-counting detectors. In a practical setting, one may wish to know whether or not position-sensitive capability is worth the added cost. This is a complex issue due to the variety of high-energy photon detection technologies. In many technologies, such as scintillators or position-sensitive Compton imaging systems, the position-sensitive capability comes at little to no extra cost because they are designed to be imaging systems. Furthermore, position-sensitive Compton imaging is an emerging technology and its current price does not reflect its true cost if it were to be mass-produced. In practice, one could use the analysis framework of this paper to perform a cost-benefit analysis of available technology.

This work focused on evaluating the detection performance of position-sensitive detectors and comparing it to the performance of counting detectors. Future work could extend this analysis to networks of position-sensitive sensors. Since counting sensors are typically much less expensive, future work will lay the foundation for cost-benefit analysis for use of position-sensitive detectors for networked applications. The numerical calculations considered single a photon energy, and future work should consider energy spectra [18]. The numerical results were for single photon interaction detectors, and future work will extend these results to Compton detectors, which can record multiple interactions. In processing Compton interactions, one often forms an approximate model for the system response, e.g., [18]. Future work to evaluate the asymptotic detection performance of Compton detectors should account for the model mismatch introduced by approximations to the system response function.

\section{APPENDIX}

\section{A. Proof of Theorem 1}

Proof: We first show the inequality (21). By the block matrix inversion formula applied to the Fisher information matrix (9),

$$
\left(\mathrm{F}^{-1}(\boldsymbol{\theta})_{[1,1]}\right)^{-1}=\mathrm{F}_{\mathrm{c}}(\boldsymbol{\theta})\left(K_{[1,1]}-K_{[2,1]}^{T} K_{[2,2]}^{-1} K_{[2,1]}\right),
$$

thus, to show (21), it suffices to show that

$$
\left(\left(K_{[1,1]}-1\right)-K_{[2,1]}^{T} K_{[2,2]}^{-1} K_{[2,1]}\right)>0 .
$$

To simplify notation, we introduce the following shorthand: $\mathrm{p}=\mathrm{p}(\boldsymbol{r} \mid D ; \boldsymbol{\theta})$ and $\mathrm{p}_{S}=\mathrm{p}_{S}(\boldsymbol{r} \mid D ; \boldsymbol{\phi})$. Since the sensitivity is uniform, let $s(\boldsymbol{\phi})=s_{0}$ for all $\boldsymbol{\phi} \in \Phi$. Now, see (28), shown at the bottom of the page. Using the fact that

$K_{[1,1]}-1=\mathrm{E}\left[\frac{\mathrm{p}_{S}^{2}}{\mathrm{p}^{2}}\right]-2 \mathrm{E}\left[\frac{\mathrm{p}_{S}}{\mathrm{p}}\right]+\mathrm{E}\left[\frac{\mathrm{p}^{2}}{\mathrm{p}^{2}}\right]=\mathrm{E}\left[\frac{\left(\mathrm{p}_{S}-\mathrm{p}\right)^{2}}{\mathrm{p}^{2}}\right]$

we can rewrite (28) as (29)-(31), shown at the top of the next page.

Equality holds when $\mathrm{p}_{S}(\boldsymbol{r} \mid D ; \boldsymbol{\phi})=\mathrm{p}(\boldsymbol{r} \mid D ; \boldsymbol{\theta})$ because in this case,

$$
\mathrm{E}\left[\left(\frac{\mathrm{p}_{S}-\mathrm{p}}{\mathrm{p}}\right)^{2}\right]=\mathrm{E}\left[\left(\frac{\mathrm{p}_{S}-\mathrm{p}_{S}}{\mathrm{p}_{S}}\right)^{2}\right]=0,
$$

and

$$
K_{[2,1]}=\mathrm{E}\left[\frac{\nabla_{\boldsymbol{\phi}}\left(s_{0} \mathrm{p}_{S}\right)}{\mathrm{p}_{S}}\right]=s_{0} \int_{\mathcal{R}} \nabla \mathrm{p}_{S}(\boldsymbol{r} \mid D ; \boldsymbol{\phi}) d \boldsymbol{r}=\mathbf{0},
$$

$$
\begin{aligned}
K_{[1,1]}-1-K_{[2,1]}^{T} K_{[2,2]}^{-1} K_{[2,1]}= & K_{[1,1]}-1-2 K_{[2,1]}^{T} K_{[2,2]}^{-1} K_{[2,1]}+K_{[2,1]}^{T} K_{[2,2]}^{-1} K_{[2,1]} \\
= & \left(K_{[1,1]}-1\right)-\mathrm{E}\left[\frac{\mathrm{p}_{S} \nabla_{\boldsymbol{\phi}}^{T}\left(s_{0} \mathrm{p}_{S}\right)}{\mathrm{p}^{2}}\right] K_{[2,2]}^{-1} K_{[2,1]}-K_{[2,1]}^{T} K_{[2,2]}^{-1} \mathrm{E}\left[\frac{\mathrm{p}_{S} \nabla_{\boldsymbol{\phi}}\left(s_{0} \mathrm{p}_{S}\right)}{\mathrm{p}^{2}}\right] \\
& +K_{[2,1]}^{T} K_{[2,2]}^{-1} \mathrm{E}\left[\frac{\nabla_{\boldsymbol{\phi}}\left(s_{0} \mathrm{p}_{S}\right) \nabla_{\boldsymbol{\phi}}^{T}\left(s_{0} \mathrm{p}_{S}\right)}{\mathrm{p}^{2}}\right] K_{[2,2]}^{-1} K_{[2,1]} .
\end{aligned}
$$




$$
\begin{aligned}
& K_{[1,1]}-1-K_{[2,1]}^{T} K_{[2,2]}^{-1} K_{[2,1]} \\
& =\mathrm{E}\left[\frac{\left(\mathrm{p}_{S}-\mathrm{p}\right)^{2}}{\mathrm{p}^{2}}\right]-\mathrm{E}\left[\frac{\left(\mathrm{p}_{S}-\mathrm{p}\right) \nabla_{\boldsymbol{\phi}}^{T}\left(s_{0} \mathrm{p}_{S}\right)}{\mathrm{p}^{2}} K_{[2,2]}^{-1} K_{[2,1]}\right]-\mathrm{E}\left[\left(\frac{\nabla_{\boldsymbol{\phi}}^{T}\left(s_{0} \mathrm{p}_{S}\right)}{\mathrm{p}} K_{[2,2]}^{-1} K_{[2,1]}\right)^{T} \frac{\mathrm{p}_{S}-\mathrm{p}}{\mathrm{p}}\right] \\
& \quad+\mathrm{E}\left[\left(\frac{\nabla_{\boldsymbol{\phi}}^{T}\left(s_{0} \mathrm{p}_{S}\right)}{\mathrm{p}} K_{[2,2]}^{-1} K_{[2,1]}\right)^{T}\left(\frac{\nabla_{\boldsymbol{\phi}}^{T}\left(s_{0} \mathrm{p}_{S}\right)}{\mathrm{p}} K_{[2,2]}^{-1} K_{[2,1]}\right)\right] \\
& =\mathrm{E}\left[\left(\frac{\mathrm{p} S-\mathrm{p}}{\mathrm{p}}-\frac{\nabla_{\boldsymbol{\phi}}^{T}\left(s_{0} \mathrm{p}_{S}\right)}{\mathrm{p}} K_{[2,2]}^{-1} K_{[2,1]}\right)^{2}\right] \\
& \quad 0 .
\end{aligned}
$$

where $\mathbf{0}$ is the zero vector, so equality is attained in (27). Note that (29) follows from (28) by the fact that when sensitivity is uniform,

$$
\mathrm{E}\left[\frac{\mathrm{p}_{S} \nabla_{\boldsymbol{\phi}}\left(s_{0} \mathrm{p}_{S}\right)}{\mathrm{p}^{2}}\right]=\mathrm{E}\left[\frac{(\mathrm{p} S-\mathrm{p}) \nabla_{\boldsymbol{\phi}}\left(s_{0} \mathrm{p}_{S}\right)}{\mathrm{p}^{2}}\right]
$$

because

$$
\mathrm{E}\left[\frac{\mathrm{p} \nabla_{\boldsymbol{\phi}}^{T}\left(s_{0} \mathrm{p}_{S}\right)}{\mathrm{p}^{2}}\right]=s_{0} \int_{\mathcal{R}} \nabla_{\boldsymbol{\phi}}^{T} \mathrm{p}_{S}(\boldsymbol{r} \mid D ; \boldsymbol{\phi}) d \boldsymbol{r}=\mathbf{0}^{T} .
$$

\section{B. Sample Derivations of Fisher Information}

Derivation of $K_{[1,1]}$ : The $[1,1]$ component of the Fisher information is given by

$$
\mathrm{F}(\boldsymbol{\theta})_{[1,1]}=-\mathrm{E}\left[\frac{\partial^{2}}{\partial \alpha^{2}} \mathrm{~L}(\boldsymbol{\theta})\right],
$$

where $L(\theta)$ is the log-likelihood, which is the logarithm of the likelihood in (4). Substituting

$$
\frac{\partial^{2}}{\partial \alpha^{2}} \mathrm{~L}(\boldsymbol{\theta})=-\sum_{i=1}^{J} \frac{\left(s(\boldsymbol{\phi}) \mathrm{p}_{S}\left(\boldsymbol{r}_{i} \mid D ; \boldsymbol{\phi}\right)\right)^{2}}{\left(\lambda_{b} \mathrm{p}_{B}\left(\boldsymbol{r}_{\boldsymbol{i}} \mid D ; \boldsymbol{\beta}\right)+\alpha s(\boldsymbol{\phi}) \mathrm{p}_{S}\left(\boldsymbol{r}_{i} \mid D ; \boldsymbol{\phi}\right)\right)^{2}},
$$

and using (3) with iterated expectation yields

$$
\begin{aligned}
\mathrm{F}(\boldsymbol{\theta})_{[1,1]} & =\frac{s^{2}(\boldsymbol{\phi})}{\left(\lambda_{b}+\alpha s(\boldsymbol{\phi})\right)^{2}} \mathrm{E}\left[\sum_{j=1}^{J} \frac{\mathrm{p}_{S}^{2}\left(\boldsymbol{r}_{i} \mid D ; \boldsymbol{\phi}\right)}{\mathrm{p}^{2}\left(\boldsymbol{r}_{i} \mid D ; \boldsymbol{\theta}\right)}\right] \\
& =\frac{s^{2}(\boldsymbol{\phi})}{\left(\lambda_{b}+\alpha s(\boldsymbol{\phi})\right)^{2}} \mathrm{E}[J] \mathrm{E}\left[\frac{\mathrm{p}_{S}^{2}(\boldsymbol{r} \mid D ; \boldsymbol{\phi})}{\mathrm{p}^{2}(\boldsymbol{r} \mid D ; \boldsymbol{\theta})}\right] \\
& =\frac{\tau s^{2}(\boldsymbol{\phi})}{\lambda_{b}+\alpha s(\boldsymbol{\phi})} \mathrm{E}\left[\frac{\mathrm{p}_{S}^{2}(\boldsymbol{r} \mid D ; \boldsymbol{\phi})}{\mathrm{p}^{2}(\boldsymbol{r} \mid D ; \boldsymbol{\theta})}\right] \\
& =\frac{\tau s^{2}(\boldsymbol{\phi})}{\lambda_{b}+\alpha s(\boldsymbol{\phi})} K_{[1,1]}=\mathrm{F}_{\mathrm{c}}(\boldsymbol{\theta}) K_{[1,1]} .
\end{aligned}
$$

Derivation of $K_{[2,1]}$ : The $[2,1]$ component of the Fisher information is given by

$$
\mathrm{F}(\boldsymbol{\theta})_{[2,1]}=-\mathrm{E}\left[\nabla_{\boldsymbol{\phi}} \frac{\partial}{\partial \alpha} \mathrm{L}(\boldsymbol{\theta})\right] .
$$

Using the model in (4),

$\mathrm{F}(\boldsymbol{\theta})_{[2,1]}$

$$
\begin{aligned}
= & \tau \nabla_{\boldsymbol{\phi}} s(\boldsymbol{\phi})-\frac{\mathrm{E}[J]}{\lambda_{b}+\alpha s(\boldsymbol{\phi})} \mathrm{E}\left[\frac{\nabla_{\boldsymbol{\phi}}\left(s(\boldsymbol{\phi}) \mathrm{p}_{S}(\boldsymbol{r} \mid D ; \boldsymbol{\phi})\right)}{\mathrm{p}(\boldsymbol{r} \mid D ; \boldsymbol{\theta})}\right] \\
& +\frac{\mathrm{E}[J] \alpha s(\boldsymbol{\phi})}{\left(\lambda_{b}+\alpha s(\boldsymbol{\phi})\right)^{2}} \mathrm{E}\left[\frac{\mathrm{p}_{S}(\boldsymbol{r} \mid D ; \boldsymbol{\phi}) \nabla_{\boldsymbol{\phi}}\left(s(\boldsymbol{\phi}) \mathrm{p}_{S}(\boldsymbol{r} \mid D ; \boldsymbol{\phi})\right)}{\mathrm{p}^{2}(\boldsymbol{r} \mid D ; \boldsymbol{\theta})}\right] \\
= & \tau \nabla_{\boldsymbol{\phi}} s(\boldsymbol{\phi})-\tau \mathrm{E}\left[\frac{\nabla_{\boldsymbol{\phi}}\left(s(\boldsymbol{\phi}) \mathrm{p}_{S}(\boldsymbol{r} \mid D ; \boldsymbol{\phi})\right)}{\mathrm{p}(\boldsymbol{r} \mid D ; \boldsymbol{\theta})}\right] \\
& +\frac{\tau \alpha s(\boldsymbol{\phi})}{\lambda_{b}+\alpha s(\boldsymbol{\phi})} \mathrm{E}\left[\frac{\mathrm{p}_{S}(\boldsymbol{r} \mid D ; \boldsymbol{\phi}) \nabla_{\boldsymbol{\phi}}\left(s(\boldsymbol{\phi}) \mathrm{p}_{S}(\boldsymbol{r} \mid D ; \boldsymbol{\phi})\right)}{\mathrm{p}^{2}(\boldsymbol{r} \mid D ; \boldsymbol{\theta})}\right] \\
= & \frac{\tau \alpha s(\boldsymbol{\phi})}{\lambda_{b}+\alpha s(\boldsymbol{\phi})} \mathrm{E}\left[\frac{\mathrm{p}_{S}(\boldsymbol{r} \mid D ; \boldsymbol{\phi}) \nabla_{\boldsymbol{\phi}}\left(s(\boldsymbol{\phi}) \mathrm{p}_{S}(\boldsymbol{r} \mid D ; \boldsymbol{\phi})\right)}{\mathrm{p}^{2}(\boldsymbol{r} \mid D ; \boldsymbol{\theta})}\right] \\
= & \frac{\alpha \mathrm{F}_{c}(\boldsymbol{\theta})}{s(\boldsymbol{\phi})} K_{[2,1]},
\end{aligned}
$$

where the third step follows because

$$
\begin{aligned}
\mathrm{E} & {\left[\frac{\nabla_{\boldsymbol{\phi}}\left(s(\boldsymbol{\phi}) \mathrm{p}_{S}(\boldsymbol{r} \mid D ; \boldsymbol{\phi})\right)}{\mathrm{p}(\boldsymbol{r} \mid D ; \boldsymbol{\theta})}\right] } \\
& =\int_{\mathcal{R}} \nabla_{\boldsymbol{\phi}} s(\boldsymbol{\phi}) \mathrm{p}_{S}(\boldsymbol{r} \mid D ; \boldsymbol{\phi}) d \boldsymbol{r} \\
& =\nabla_{\boldsymbol{\phi}} s(\boldsymbol{\phi}) \int_{\mathcal{R}} \mathrm{p}_{S}(\boldsymbol{r} \mid D ; \boldsymbol{\phi}) d \boldsymbol{r}=\nabla_{\boldsymbol{\phi}} s(\boldsymbol{\phi}),
\end{aligned}
$$

by the assumption that integration and differentiation are interchangeable.

The other terms in (9) have similar derivations.

\section{REFERENCES}

[1] R. C. Runkle, T. M. Mercier, K. K. Anderson, and D. K. Carlson, "Point source detection and characterization for vehicle radiation portal monitors," IEEE Trans. Nucl. Sci., vol. 52, no. 6, pp. 3020-3025, 2005.

[2] P. C. Schaich, G. A. Clark, S. K. Sengupta, and K. P. Ziock, "Automatic image analysis for detecting and quantifying gamma-ray sources in coded-aperture images," IEEE Trans. Nucl. Sci., vol. 43, no. 4, pp. 2419-2426, 1996.

[3] Z. Liu and A. Nehorai, "Detection of particle sources with directional detector arrays and a mean-difference test," IEEE Trans. Signal Process., vol. 53, no. 12, pp. 4472-4484, 2005.

[4] Z. He, W. Li, G. F. Knoll, D. K. Wehe, J. Berry, and C. M. Stahle, "3-D position sensitive CdZnTe gamma-ray spectrometers," Nucl. Instrum. Methods Phys. Res. A, Accel., Spectrom., Detect. Assoc. Equip., pp. 173-178, Feb. 1999. 
[5] K. Vetter, "Gamma-ray imaging with position-sensitive HPGE detectors," Nucl. Instrum. Methods Phys. Res. A, Accel., Spectrom., Detect. Assoc. Equip., vol. 525, no. 1-2, pp. 322-327, Jun. 2004.

[6] C. G. Wahl and Z. He, "Sensitivity of gamma-ray source detection using 3D-position-sensitive semiconductor detectors," in Proc. IEEE Nuclear Science Symp. Conf. Rec. (NSS), 2008, pp. 3334-3338.

[7] K. P. Ziock, W. W. Craig, L. Fabris, R. C. Lanza, S. Gallagher, B. K. P. Horn, and N. W. Madden, "Large area imaging detector for long-range, passive detection of fissile material," IEEE Trans. Nucl. Sci., vol. 51, no. 5, pp. 2238-2244, 2004.

[8] H. H. Barrett, T. White, and L. C. Parra, "List-mode likelihood," J. Opt. Soc. Amer. A, vol. 14, no. 1, pp. 2914-23, Nov. 1997.

[9] J. A. Hanley and B. J. McNeil, "The meaning and use of the area under a receiver operating characteristic (ROC) curve," Radiol., vol. 143, no. 1, pp. 29-36, Apr. 1982.

[10] F. O'Sullivan, "Imaging radiotracer model parameters in PET: A mixture analysis approach," IEEE Trans. Med. Imag., vol. 12, pp. 399-412, Sep. 1993.

[11] S. Vallabhajosula, Molecular Imaging: Radiopharmaceuticals for PET and SPECT. Berlin, Germany: Springer-Verlag, 2009.

[12] S. M. Kay, Fundamentals of Statistical Signal Processing: Detection Theory. New York: Prentice-Hall, 1998.

[13] R. Protassov, D. A. van Dyk, A. Connors, V. L. Kashyap, and A. Siemiginowska, "Statistics: Handle with care, detecting multiple model components with the likelihood ratio test," Astrophys. J., vol. 571, pp. 545-559, Jan. 2002.

[14] Z. Liu and A. Nehorai, "Statistical angular resolution limit for point sources," IEEE Trans. Signal Process., vol. 55, no. 11, pp. 5521-5527, Nov. 2007

[15] R. E. Bethel and K. L. Bell, "Maximum likelihood approach to joint array detection/estimation," IEEE Trans. Aerosp. Electron. Syst., vol. 40, no. 3, pp. 1060-1072, Sep. 2004

[16] G. F. Knoll, Radiation Detection and Measurement, 2nd ed. New York: Wiley, 1989.

[17] D. Feldman and M. Fox, Probability: The Mathematics of Uncertainty. New York: Marcel Dekker, 1991.

[18] D. Xu and Z. He, "Gamma-ray energy-imaging integrated spectral deconvolution," Nucl. Instrum. Methods Phys. Res. A, Accel. Spectrom. Detect. Assoc. Equip., vol. 574, no. 1, pp. 98-109, Apr. 2007.

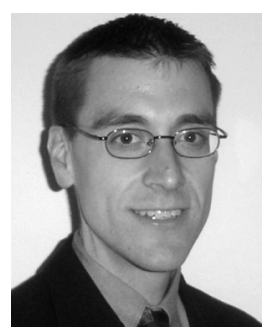

Daniel J. Lingenfelter (S'08) received the B.S.E. degree in electrical engineering and the M.S.E. degree in electrical engineering systems, both from the University of Michigan, Ann Arbor, in 2006 and 2009, respectively.

$\mathrm{He}$ is currently a graduate student in the Department of Electrical Engineering and Computer Science at the University of Michigan, Ann Arbor. His research interests include statistical signal processing, particularly imaging and detection theory.

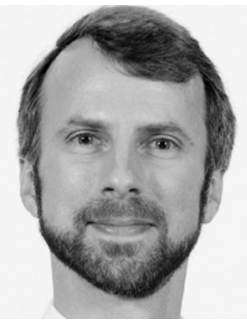

Jeffrey A. Fessler (S'90-M'90-SM'00-F'06) received the B.S.E.E. degree from Purdue University, West Lafayette, IN, in 1985 and the M.S.E.E. degree and the M.S. degree in statistics from Stanford University, Stanford, CA, in 1986 and 1989, respectively. In 1990, he received the Ph.D. degree in electrical engineering from Stanford University, where he was a National Science Foundation Graduate Fellow.

Since then he has worked at the University of Michigan, Ann Arbor. From 1991 to 1992, he was a Department of Energy Alexander Hollaender Post-Doctoral Fellow in the Division of Nuclear Medicine. From 1993 to 1995, he was an Assistant Professor in Nuclear Medicine and the Bioengineering Program. He is now a Professor in the Departments of Electrical Engineering and Computer Science, Radiology, and Biomedical Engineering. His research interests are in statistical aspects of imaging problems, and he has supervised doctoral research in PET, SPECT, X-ray CT, MRI, and optical imaging problems.

Dr. Fessler received the Francois Erbsmann award for his IPMI93 presentation. He serves as an Associate Editor for the IEEE TRANSACTIONS ON MEDICAL IMAGING and is a past Associate Editor for the IEEE TRANSACTIONS ON IMAGE PROCESSING and the IEEE SigNAL PROCESSING LeTTERS. He was Co-Chair of the 1997 SPIE Conference on Image Reconstruction and Restoration, Technical Program Co-Chair of the 2002 IEEE International Symposium on Biomedical Imaging (ISBI), and General Chair of the ISBI 2007.

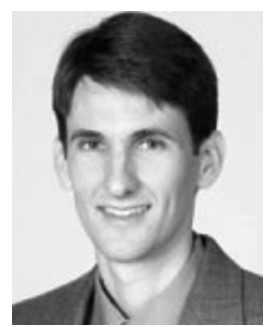

Clayton D. Scott (S'99-M'04) received the A.B. degree in mathematics from Harvard University, Cambridge, MA, in 1998 and the M.S. and Ph.D. degrees in electrical engineering from Rice University, Houston, TX, in 2000 and 2004, respectively.

He was a Postdoctoral Fellow in the Department of Statistics at Rice University and is currently an Assistant Professor in the Departments of Electrical Engineering and Computer Science and of Statistics at the University of Michigan, Ann Arbor. His research interests include pattern recognition, machine learning, statistical signal processing, and applications.

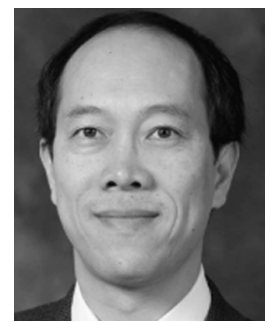

Zhong He (M'98-SM'00) received the B.S. degree in physics from Tsinghua University and the M.S degree at the High Energy Physics Institute, Beijing, China, and the Ph.D. degree from Southampton University, U.K., in 1993.

Since 1994, he has worked at the University of Michigan, Ann Arbor, where he worked initially as a Research Scientist and then became a teaching faculty member in 1998. His research during 1986 to 1995 focused on scintillation detectors, such as NaI, CsI, BGO, LSO, coupled with position-sensitive PM tubes, or silicon photodoides. Since 1995, his research has focused mostly on the development of coplanar-grid and 3-dimensional position-sensitive wide bandgap semiconductor gamma-ray imaging spectrometers. He pioneered the 3-dimensional position-sensitive single polarity charge sensing technology which has received increasing interests in homeland security, nuclear nonproliferation, astrophysics and planetary sciences. He has also worked on high-pressure Xe gamma-ray detectors. His research has covered all three major types of radiation sensors, semiconductor, scintillation and gas detectors. Since 1999, his group has developed very low noise, room-temperature operation charge sensing application specific integrated circuitries (ASICs) in collaboration with Gamma-Medica-Ideas AS, Norway, and Brookhaven National Laboratory to measure signal amplitude and charge drift-time simultaneously in semiconductors. He has graduated nine Ph.D. candidates. 